\title{
Correction to: Automated IMRT planning in Pinnacle-A study in head-and-neck cancer
}

\author{
J. M. A. M. Kusters ${ }^{1}$ K. Bzdusek ${ }^{2}$ P. Kumar ${ }^{3}$ P. G. M van Kollenburg ${ }^{1}$ M. C. Kunze-Busch ${ }^{1}$. \\ M. Wendling ${ }^{1}$ T. Dijkema ${ }^{1}$ J. H. A. M. Kaanders ${ }^{1}$
}

Published online: 27 October 2017

(C) Springer-Verlag GmbH Deutschland 2017

\section{Correction to:}

\section{Strahlenther Onkol 2017}

https://doi.org/10.1007/s00066-017-1187-9

Unfortunately, parts of the 'Materials and Methods section' and a sentence in the 'Discussion section' had to be corrected.

On page 3, left column, the complete first paragraph was corrected and now reads as follows:

Auto-Planning is fully integrated into Pinnacle v.9.10 TPS to automate the inverse planning process [11-13].

On page 3, left column, the complete third paragraph was corrected and now reads as follows:

Thus, Auto-planning is designed to automatically perform many of the manual operations in the IMRT planning process.

On page 3, right column, second paragraph to page 4 left column, first paragraph, the complete section was corrected and now reads as follows:

Table 2 shows the Auto-Planning optimization preset used for the patients in this study. In the Advanced Setting Tab (see also Ref. 20) several additional parameters

The online version of the original article can be found at https:// doi.org/10.1007/s00066-017-1187-9.

$\triangle$ J. M. A. M. Kusters, PhD

martijn.kusters@radboudumc.nl

1 Department of Radiation Oncology, Radboud university medical center, Postbox 9101, 6500 HB Nijmegen, The Netherlands

2 Philips Radiation Oncology Systems, Philips Healthcare, Fitchburg, WI 53711, USA

3 Philips Innovation Campus, Philips Electronics India Ltd., Bangalore, India can be defined. "The Tuning Balance" adjust the balance between the competing goals of target conformity and OAR sparing. This value was set to $10 \%$, to obtain a highly conformal dose in the PTV. The "Dose Fall-Off Margin" is a margin around targets for dose fall-off. In this region, the Auto-Planning tool makes stronger attempts to reduce dose. "The Hot-Spot Maximum Goal" can be set as a percentage with respect to the maximum prescribed dose to the target and will be used to reduce the maximum dose of hotspots in the target. By selecting "Use Cold-Spot ROIs", the AutoPlanning tool creates ROIs for any cold spots lower than the prescribed dose within the target and creates objectives for those cold-spot ROIs to further optimize the target dose coverage. When biological optimization is selected, the AutoPlanning uses EUD objectives in case the mean dose goals are selected. It uses MAX EUD objectives with an "a" value of 1 to achieve mean dose goals. There is no TCP/NTCP or varied "a" values. The following three goal types can be used, Maximum dose, Mean dose, and Max DVH dose. The conventional weight factors in Pinnacle can not been set in the Auto-planning tool and priority of the goal can be set by Low, Medium or High. The option to put a hard constraint on the goal is suitable, but would not lead to better results from our experience. The last column for the OAR goals in Table 2 shows our setting of the Compromise option. With "Compromise" set to "YES" the overlapping region of an OAR with a target will be assigned to the target.

On page 4, left column, the complete second paragraph was corrected and now reads as follows:

The Auto-Planning tool can automatically adjust the priority values of OAR optimization goals based on ROI overlap statistics. If the ROI used in an OAR optimization goal substantially overlaps any targets, the Auto-Planning tool lowers its priority. 
In the 'Discussion section' on page 6 , right column, the second paragraph was corrected and now reads as follows:

To get the Auto-Planning optimization preset, a certain amount of effort has to be made to find the optimal setting for the objectives. The use of Scorecards in Pinnacle is recommended to check if the planning goals are met. 